\title{
Thoughts about the Ventricular Gradient and Its Current Clinical Use (Part I of II)
}

\author{
J. WILLIS HURST, M.D., MACP \\ Emory University, Atlanta, Georgia, USA
}

Summary: The concept of the ventricular gradient was conceived in the mind of Frank Wilson in the early 1930s. Wilson, a mathematical genius, believed that the calculation of the ventricular gradient yielded information that was not otherwise obtainable. The method of analysis was not utilized by clinicians at large because the concept was not easy to understand and because the method used to compute the direction of the ventricular gradient was so time consuming that clinicians could not use it. Grant utilized the concept to create vector electrocardiography, but he believed that if his method of analysis was used, it was not often necessary to compute the direction of the ventricular gradient. He did, however, describe an easy way to compute the direction of the ventricular gradient. The current major clinical use of the ventricular gradient is to identify primary and secondary T-wave abnormalities in an electrocardiogram showing left or right ventricular hypertrophy or left or right ventricular conduction abnormalities. In addition, the author uses the term ventricular time gradient instead of ventricular gradient in an effort to clarify the concept. Finally, the author discusses the possible clinical significance of a normally directed but shorter than normal ventricular time gradient, an attribute that has not been emphasized previously.

Key words: ventricular time gradient

\section{Introduction}

Wilson's classic communication, coauthored by Macleod, Barker, and Johnston, entitled "The Determination and the

\section{Address for reprints:}

J. Willis Hurst, M.D., MACP

Emory University

1462 Clifton Road, N.E.

Suite 301

Atlanta, GA 30322, USA

e-mail: jhurst@emory.edu

Received: February 4, 2005

Accepted: February 4, 2005
Significance of the Areas of the Ventricular Deflections of the Electrocardiogram" was published in 1934. ${ }^{*}$

They wrote:

It is the purpose of this article to describe a method of analyzing the electrocardiogram which has not been employed heretofore and which yields information not obtainable in other ways. ${ }^{1}$

Except for Bayley and Monti, ${ }^{2}$ Wilson's concept, later called the ventricular gradient, was understood by only a few researchers and even fewer clinicians. ${ }^{2}$

Ashman and his colleagues wrote four masterful articles on the subject in $1943 .^{3-6}$ They hoped to interest others in the ventricular gradient, but their well-written articles produced no ground swell of interest in the subject.

Burch and Winsor wrote about the subject in their book $A$ Primer of Electrocardiography in $1945 .{ }^{7}$ Even though their illustrations were superb and their description of the gradient was perhaps the clearest ever written, they failed to stimulate clinicians to use the ventricular gradient in their daily work.

Finally, even Barker, who was one of the authors of Wilson's original paper, ${ }^{1}$ seemed to lose interest in the subject. In 1952, in his book The Unipolar Electrocardiogram he wrote,

At the present time, the determination of the ventricular gradient is not a practical procedure for general use in electrocardiography. The theoretical foundations upon which it rests, however, are of utmost importance. ${ }^{8}$

Grant developed vector electrocardiography in the late 1940s and early 1950s. His classic book, Clinical Electrocardiography, was published in $1957 .{ }^{9}$ Grant's work, which was based on Wilson's concepts, made it possible to use vector concepts in the routine analysis of tracings. In fact, his method incorporates certain aspects of the gradient itself and makes it possible to determine the direction of the ventricular gradient when such a computation is needed. Even he, how-

\footnotetext{
*Wilson et al. added this footnote to their article: "A preliminary report based upon the material presented in the article cited as reference 1 was published in the Proc Soc Exper Biol \& Med 1930; 27:591. See also Wilson, Macleod, and Barker: Tr Assoc Am Physicians 1931;46:29."
} 
ever, rightfully insisted that it was not necessary to calculate the gradient in every tracing, especially if his method of analysis was used. ${ }^{9 a}$

The purpose of this article is to define and explain the ventricular gradient and to discuss when the calculation of its direction and size is of clinical value today.

\section{Definition of the Words}

The word ventricular is understood but the word gradient confuses many interpreters of electrocardiograms. The meaning of the word is best understood by citing examples in which a gradient exists. Suppose the slope of a road going up a mountain has an altitude gradient of 100 feet. The measurement is made from the lowest part of the road to the highest part of the road. Suppose the peak systolic pressure gradient across an aortic valve in a patient with aortic valve stenosis is $100 \mathrm{~mm}$ of mercury. This measurement is made by subtracting the peak systolic pressure in the aorta (which is $100 \mathrm{~mm}$ of mercury) from the peak systolic pressure in the left ventricles which is $200 \mathrm{~mm}$ of mercury. The difference in pressures is called a pressure gradient.

When a cell loses all of its electrical charges, it is considered to be depolarized (there are no electrical charges on the cell). The depolarized cell is said to be excited. This is followed by a period of electrical inactivity, which is followed by repolarization (the restoration of electrical charges). When all parts of the cell are able to restore the electrical charges after the same amount of time has elapsed, there is no time gradient. On the other hand, when a part of the cell restores its electrical charges following a delay in time that is greater than the time required for the rest of the cell to restore its electrical charges, a time gradient is produced. This was called a T-altering force or a Tdelaying force by Grant. ${ }^{\mathrm{b}}$ Stated another way, the ventricular gradient is the mathematical symbol that illustrates the variation of the duration of the excited state of a cell, or a group of cells (including the entire ventricular muscle).

This simply stated preamble is added here with the hope it will prepare the reader for the remainder of the discussion in which the details of the ventricular time gradient and its current clinical use are described.

\section{Required Background Information}

\section{A Hypothetical Cell}

Many workers in the field have described and illustrated the electrical behavior of a single, isolated, hypothetical myocardial cell, but none have done it better than Burch and Winsor. ${ }^{10}$ The discussion and diagrams used in this section are based on their discussion of the subject. ${ }^{10}$

The reader must remember that the following text and illustrations deal with the events taking place in a hypothetical cell. Accordingly, the discussion and figures do not accurately portray the events, but are designed to teach a very complicated process which is often ignored because of its complexity. For example, the depolarization and repolarization of a single cell cannot create an electrocardiogram as shown in the figures. Also, the arrangement of the electrical charges created by ions inside and outside the cell membrane is far more complex than shown here. Still, the imperfect discussion and figures teach a concept that is otherwise difficult to grasp by busy clinicians.

It is useful to imagine a single cell that is shaped like a sphere and has a surface membrane that is endowed with the innate ability to create electrical charges. Negative charges are located on the inner surface of the cell membrane and positive charges are located on the outside of the cell membrane (Fig. 1). Note that each negative charge is matched with a positive charge. In your mind, you should perceive that each of the matched charges creates a small arrow that represents an electrical force. Note too that there is an imagined measuring device that records the electrical activity of the cell. The measuring device records an upright deflection when the sum of all of the electrical forces is directed toward the positive pole of the machine and a negative deflection when the sum of all the electrical forces is directed away from the positive pole of the machine.

Although a saggital view of the cell is shown in the figure, one must remember that the cell is spherical in shape and that the charges (and arrows) are located on all parts of the cell membrane. A three-dimensional cell such as this is considered to be a polarized or resting cell.

\section{The Process of Depolarization}

Suppose the right side of the cell is stimulated by an electrical source that originates outside the cell. This causes the electrical charges to vanish from that part of the cell that is

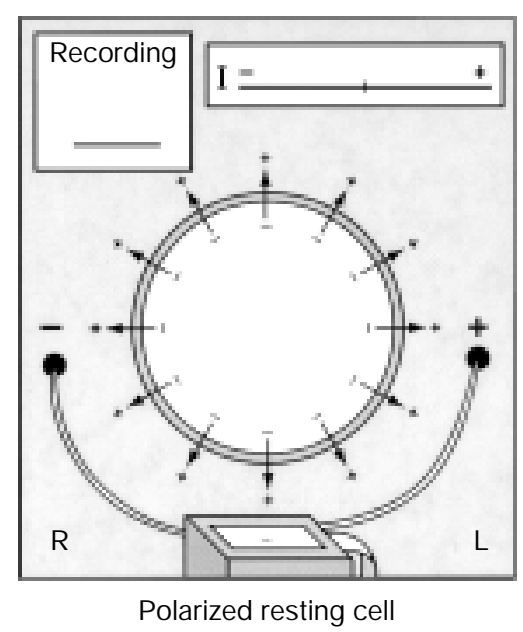

FIG. 1 A hypothetical cell. It should be considered as being shaped like a sphere. The measuring device itself is shown below. The negative pole of the measuring device is located on the right and the positive pole of the measuring device on the left. The recording of the deflection is shown in "lead I" in the upper left corner. 
stimulated initially (Fig. 2A). The cell loses its charges in an orderly manner that is referred to as depolarization. This causes the measuring device to record an upright deflection. When one-half of charges vanishes, the deflection would reach its greatest amplitude (Fig. 2B). When about three-fourths of the charges vanish, the deflection would exhibit less amplitude, but would remain upright (or positive) as shown in Figure 2C. When all of the charges are gone, the cell is said to be completely depolarized or excited (Fig. 2D). The recorded upright deflection will remain in the wake of depolarization until repolarization begins.

Note that the process of depolarization is directed from right to left, creating an electrical force that is also directed from right to left. Note also that the deflection in the recording is directed upward.

\section{The Process of Repolarization after a Uniform Time Lag}

After a period of time, during which the recording devise records no electrical activity, the electrical charges begin to reappear on the cell membrane. If one assumes that all parts of the cell are equally capable of restoring its electrical charges after the same period of time delay, then the restoration of charges will occur on the cell where the charges were initially lost. Accordingly, as shown in Figure 3A, the restoration of electrical charges will begin on the right side of the cell where they initially vanished.

Figure $3 \mathrm{~B}$ shows the repolarization process at the halfway point, Figure $3 \mathrm{C}$ shows the repolarization process at the threequarter point, and Figure 3D shows the completion of the repolarization process. The cell is now completely repolarized and is resting.

Note that the repolarization process is directed from right to left, creating an electrical force that is directed from left to right. Note also that the deflection in the recording is directed downward.

This simple series of figures should be studied carefully. For example, they show that the area subtended under the upright deflection is positive and represents a crude QRS complex. It is equal in size to the area subtended under the negative deflection which represents a crude T wave. Figure 3 shows that the vectors representing the $\mathrm{QRS}$ complex and $\mathrm{T}$ wave are equal in
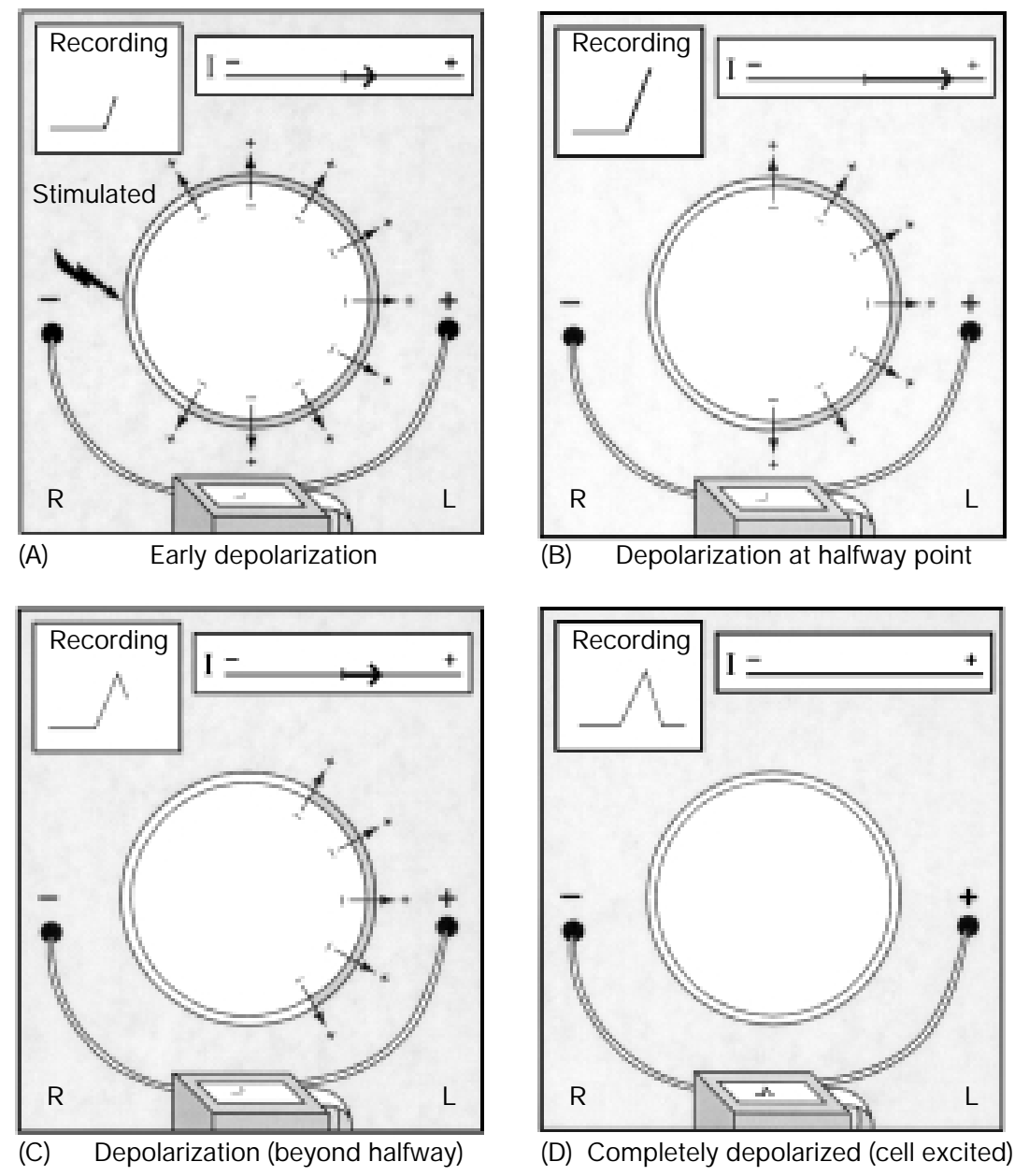

(D) Completely depolarized (cell excited)

FIG. 2 (A-D) See text and legend in Figure 1. 

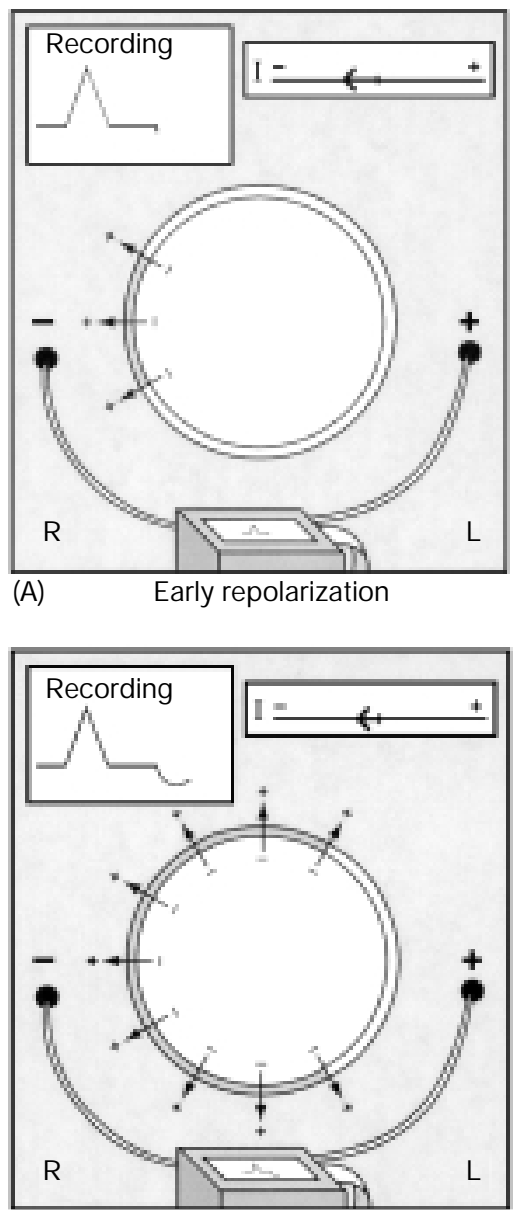

(C) Repolarization nearing completion
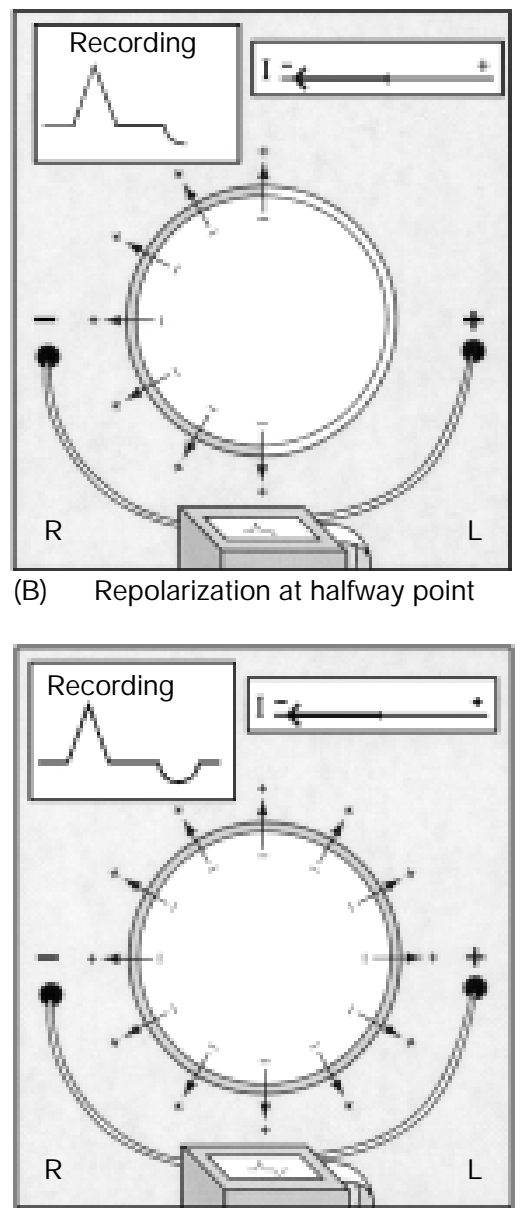

(D) Repolarization complete (begins resting state)

FIG. 3 (A-D) See text and legend in Figure 1.

size, but are directed opposite to each other. The vector sum of the two is zero. Under these circumstances, the depolarization process predetermines the direction of the repolarization process because the restoration of electrical charges is delayed after the same length of time. Since the charges are restored after the same time delay in all parts of the cell, there would be no time gradient.

\section{The Process of Repolarization after a Nonuniform Time Lag}

The following discussion shows what can happen when we tamper with the membrane of the isolated hypothetical cell. Suppose the right side of the cell is cooled and the coolest point is located as shown in Figure 4A. The depolarization process, that begins when an outside stimulus is applied to the right side of the cell, would not necessarily be interfered with and would proceed as shown in Figure 2A through D. However, the repolarization process would be influenced considerably by the cooling. The restoration of charges on the right side of the cell would be abnormally delayed to the degree that the rebuilding of charges would begin on the left side of the cell. This profoundly changes the direction of the repolarization process (Fig. 4B and C). Note that the repolarization process begins on the left side of the cell and that the process of restoring the charges is directed from left to right, creating an electrical force that is directed from right to left. This produces an upright $\mathrm{T}$ wave. The sum of the electrical forces for the depolarization of the cell (a crude QRS complex) and the sum of the electrical forces for the repolarization of the cell (a crude $\mathrm{T}$ wave) can be viewed as vectors and are represented by arrows. When this is done, the direction and size of the vectors representing depolarization and repolarization are identical (Fig. 5). The ventricular time gradient would be twice as long as the vectors representing depolarization and repolarization. The gradient is actually computed by adding the mean vector representing all of the electrical forces produced by depolarization and the mean vector representing all of the electrical forces produced by repolarization. This is accomplished by forming a parallelogram using the QRS vector and T vector as its sides; the diagonal of the figure is the ventricular gra- 


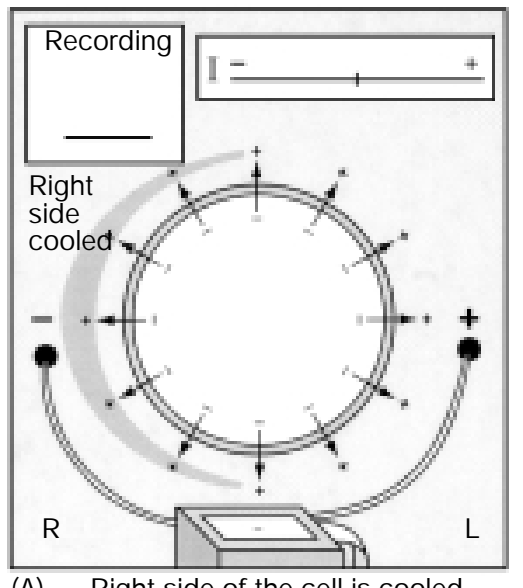

(A) Right side of the cell is cooled

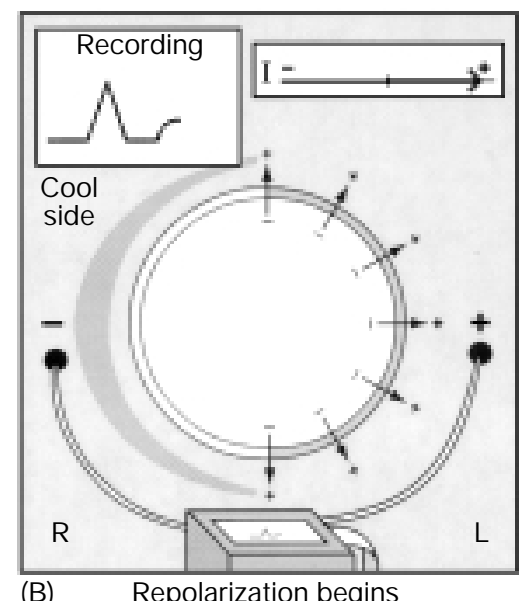

(B) Repolarization begins

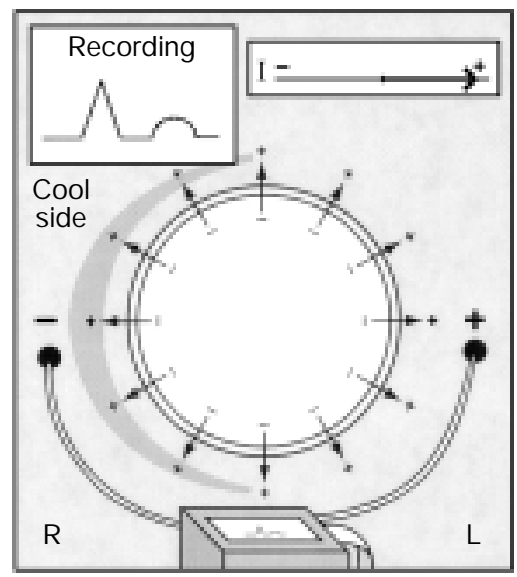

(C) Repolarization complete (begins resting state)

FIG. 4 (A-C) See text and legend in Figure 1.

dient. The ventricular time gradient, represented as a vector, is three-dimensional just as the QRS and T wave vectors are three-dimensional.

Notice in Figure 4B-C that the excited part of the cell persists longer than it does in Figure 2. This teaches, as Burch and Winsor pointed out so clearly, "that a vector representing the gradient points in a direction from a point in the cell in which the duration of the excited state is longest to that in which it is shortest."'10a

\section{What Creates the Ventricular Gradient in the Human Heart?}

It is accepted that the depolarization process of the ventricles begins, for the most part, at the endocardium and is directed toward the epicardium, creating an electrical force that is directed in the same direction. It is also accepted that the repolarization process of the ventricles begins, for the most part, in the ventricular epicardium and is directed toward the endocardium producing an electrical force that is directed in the opposite direction. The latter is reminiscent of the electrical activity of the hypothetical cell that is cooled on the right side (Fig. 4). The question then arises as to why this occurs in the heart of a normal adult! Grant taught that the QRS complex, which is

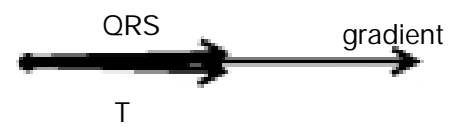

FIG. 5 When the mean QRS vector and mean T vector are located in the same direction and are equally large, the ventricular gradient will be twice as large as either of them. When the mean QRS vector is located in a certain direction and the mean $\mathrm{T}$ vector is of equal size and is located exactly opposite to the direction of the mean $Q R S$ vector, the ventricular gradient will be zero. created by depolarization, develops early in ventricular systole when the pressure in the ventricles is just developing, whereas the $\mathrm{T}$ wave is produced later in ventricular systole after the right and left ventricular peak pressure has passed, but when the intraventricular pressure is much higher than it was when the QRS complex was created. ${ }^{11} \mathrm{He}$ also taught that the pressure was higher in the endocardium than in the epicardium and that this delayed the restoration of electrical charges in the endocardial area of the heart. ${ }^{11} \mathrm{He}$ thought of this phenomenon as a transmyocardial pressure gradient. ${ }^{11}$ Therefore, the repolarization process is forced to begin in the epicardium rather than the endocardium. This, of course, reveals that the normal adult human heart creates a normal ventricular time gradient each time it contracts. The gradient is directed away from the area of the heart where there is a delay in the repolarization process. However, this concept must not be construed to contradict the fact that the direction of the repolarization process (the $\mathrm{T}$ ) is predetermined by the direction of the depolarization process (the QRS) because it is, although somewhat indirectly.

Whereas the foregoing explanation of the mechanism that is responsible for the ventricular time gradient in the adult human heart is plausible, it does not answer all of the questions. If the mechanism just described is correct then why is there a QRS-T angle in a normal heart? The answer might lie in the concept that the transmyocardial pressure gradient is not uniform throughout the ventricular myocardium.

\section{How to Determine the Directions and Size of the Ventricular Gradient}

Grant proposed a simple method for determining the spatial direction and size of the electrical forces that create the deflections in the electrocardiogram. ${ }^{11 \mathrm{a}} \mathrm{He}$ pointed out that the frontal plane direction of the mean $\mathrm{QRS}$ and T vectors could be determined instantly by inspecting the routine 12-lead electrocardiogram. He taught that when an electrical force was 
parallel to a given extremity lead axis, it produced its largest deflection in that lead, and when an electrical force was perpendicular to a given extremity lead axis it produced its smallest deflection in that lead. ${ }^{11 a}$ The area subtended by the part of the tracing being studied should be used to make the calculation. Accordingly, when the mean QRS and mean T-wave vectors are being studied, the net area subtended by the entire QRS and T waves is used to determine the size of the deflection. Therefore, it is necessary to subtract the area subtended by the part of the deflection that is below the baseline from the area subtended by the part of the tracing above the baseline. Using Grant's method, it is possible to determine the frontal plane direction of the mean QRS and T waves with acceptable accuracy. Grant also created the method used for determining the spatial direction of the electrical forces produced by the electrical activity of the heart. He pointed out that the anteroposterior direction of the frontal plane projection of an electrical force could be determined by using the transitional complex in the chest leads to estimate how far the electrical force was directed anteriorly or posteriorly. ${ }^{11 \mathrm{a}}$ This is possible because the transitional complex identifies the transitional pathway which is the body surface extension of the zero potential plane. Since the zero potential plane is perpendicular to the electrical force that produces it, it is possible to determine the approximate number of degrees an electrical force is directed anteriorly or posteriorly.

The frontal plane direction and size of the ventricular time gradient can be determined by completing the parallelogram using the QRS and T vectors as its sides. The diagonal is the ventricular time gradient. The ability to do this is why we must reconsider the value of calculating the ventricular time gradient in the interpretation of electrocardiograms. The spatial direction of the ventricular time gradient can also be calculated, but it is not nearly as precise as the frontal plane calculation of the gradient. This is because the size of the precordial lead deflections, which are used to make the calculation, is influenced by the nearness of the electrodes to the heart itself.

In the beginning, Wilson et al. enlarged the tracings and used a planimeter to measure the area subtended by the total QRS complexes and T waves in two bipolar extremity leads. ${ }^{1}$ This, of course, requires considerable time and is not practical for clinical usage. Others estimated the area subtended by the QRS complex and T waves by multiplying one half of the duration of a deflection by the amplitude of the deflection; even this is too time consuming. Grant's method of interpretation by simple inspection of the net areas of QRS and T waves is usually adequate to make the calculation.

As will be discussed subsequently, it is not always necessary to establish the size or the ventricular gradient. This is true because at times the direction of the gradient is clearly abnormal. There are times, however, when the size of a normally directed ventricular gradient is important. See later discussion.

\section{Acknowledgment}

The figures shown in Part I of this manuscript are modifications of many previously published illustrations. For example, Burch and Winsor in Reference 7 offered such illustrations, as did Hurst in Hurst JW: Ventricular Electrocardiography, p. 3.5-3.8, New York: Gower Medical Publishing, 1991.

\section{References (Part I)}

1. Wilson PF, MacLeod AG, Barker PS, Johnston FD: The determination and the significance of the areas of the ventricular deflections of the electrocardiogram. Am Heart J 1934;10:46

2. Bayley RH, Monte LA: Acute local ventricular ischemia, or impending infarction, caused by dissecting aneurysm: Case report with necropsy. Am Heart J 1943;25:262-270.

3. Ashman R, Byer E: The normal human ventricular gradient. I. Factors which affect its direction and its relation to the mean QRS axis. Am Heart $J$ 1943;25:16-35

4. Ashman R, Byer E: The normal human ventricular gradient. II. Factors which affect its manifest area and its relationship to the manifest area of the QRS complex. Am Heart J 1943;25:36-57

5. Ashman R, Gardberg M, Byer E: The normal human ventricular gradient. III. The relation between the anatomic and electrical axes. Am Heart J 1943;25:473-494

6. Ashman R: The normal human ventricle gradient. IV. The relationship between the magnitudes, AQRS and G, and deviations of the RS-T segment. Am Heart J 1943;25:495-510

7. Burch G, Winsor T: A Primer of Electrocardiography, pp. 179-189. Philadelphia: Lea \& Febiger, 1945

8. Barker JM: The Unipolar Electrocardiogram: A Clinical Interpretation, p. 578. New York: Appleton-Century-Crofts, Inc., 1952

9. Grant RP: Clinical Electrocardiography: The Spatial Vector Approach.pp. 1-225. New York: The Blakiston Division, McGraw-Hill Book Company, Inc., 1957; (a) pp. 132-134; (b) pp. 71-72

10. Burch G, Winsor T: A Primer of Electrocardiography. Philadelphia: Lea \& Febiger, 1945;23-31; (a) p. 181

11. Grant RP: Clinical Electrocardiography: The Spatial Vector Approach. New York: The Blakiston Division, McGraw-Hill Book Company, Inc., 1957:69; (a) p. 31

Part II of this paper will appear in the May issue of Clinical Cardiology. 